of glycerides to fatty acid and glycerol, followed by re-synthesis in the intestinal cell to phospholipids (as an essential intermediate), is no longer tenable.

The contributions of Dr. G. Popják and Dr. S. J. Folley on the synthesis of fatty acids in animals, especially in their mammary glands, from acetate (or from glucose via acetate or pyruvate) will receive much notice, since the recent important studies of both authors in this field are collected and discussed in relation to results obtained by other workers. It is of great advantage to have the results which have been disseminated in a large number of individual papers in the scientific journals brought together by two of the most prominent investigators of this subject. Dr. Popják's review concerns the evidence, based on work with radioactive acetate, of synthesis of fats in general from small (two-carbon) molecules, while Dr. Folley's is more specifically devoted to the biosynthesis of milk fat in ruminant mammals. The two articles together give a complete account of the subject as it stands at present.

Dr. A. L. Lehninger gives in some detail an account of recent work by himself and his colleagues on the mechanism of the enzymatic oxidation of fatty acids. The discussion includes sections on the properties of the fatty-acid oxidase system of animal tissues, on the mechanism of acetoacetate formation and oxidation, and on the significance of recent work on fatty-acid oxidation and synthesis in extracts of Clostridium kluyveri.

The final paper, by Dr. H. M. Sinclair, deals with the fatty acids (linoleic, linolenic, arachidonic) essential to growth and their relation to pyridoxine. This very interesting contribution, which merits careful study, brings out an analogy between deficiency of essential fatty acids and deficiency of pyridoxine. It is suggested that failure of growth in essential fatty-acid deficiency may be connected with inability to form the phospholipids necessary for cell membranes, and also that the essential polyunsaturated acids may be concerned in controlling the water retention of animal tissues.

It is to be hoped that this, necessarily brief, notice of the contents of the volume may serve to indicate its usefulness to all interested in the role of lipids in biochemical processes.

\section{COSMIC RAYS AND THE CLOUD CHAMBER}

\section{Cloud Chamber Photographs of the Cosmic Radia- tion}

By G. D. Rochester and J. G. Wilson. Pp. viii +128. (London: Pergamon Press, Ltd., 1952.) $70 \mathrm{~s}$.

THE authors of this book, Dr. G. D. Rochester and Prof. J. G. Wilson, are to be congratulated on its production; in bringing together in one volume a very fine collection of photographs, they have paid worthy tribute to the part the cloud chamber has played in revealing the nature and associated properties of the fundamental particles which are found in the cosmic radiation. To each photograph (the total number of which is one hundred and twenty-three) the authors have prepared a most useful synopsis giving not only the details of the actual chamber and the geometry of the accessory equipment such as position and size of lead plates, but also an analysis of the physics of the event in question; this includes both the results of the measurements made upon the tracks and a discussion of the resulting interpretation of the event. It cannot be stressed too strongly that all nuclear physicists will benefit from a study of the photographs whether or not they are versed in the technique of the use of expansion chambers. It is true that to those who are so versed the book will be of extra value in that it shows the results obtained from chambers covering a great range of sizes, operating pressures, etc.; but the outstanding merit of the book is the fact that it describes and discusses, in an interesting manner, real fundamental physics. The quality of the reproduction of the photographs is excellent and is immediately impressive.

The photographs are grouped in six sections. Section 1 is entitled "Technical Features of Operation" and illustrates the process of formation of drops, the efficiency of condensation upon ions, the effects of turbulence and contamination within a chamber, and other features. Section 2 deals with electrons and cascade showers, and the first few plates show the tracks which revealed the existence of the positron and the cascade process; photographs of extensive showers show not only the presence of cascade particles, but also penetrating particles together with nuclear interactions associated with such showers. Section $\mathbf{3}$ is concerned with the existence and subsequent decay of the $\mu$-meson; a most important group of photographs demonstrates the way in which the mass of a particle may be determined from momentum and range measurements, and includes an indication of the nature and magnitude of the uncertainties involved.

Nuclear disintegrations and interactions of secondary particles form Section 4, which covers reactions observed in multi-plate chambers (for example, of carbon and lead), as well as stars produced in the gas of the chamber.

The photographs which have made it necessary to postulate the existence of charged and uncharged $V$-particles are shown in Section 5. The interpretation of these photographs is clearly discussed and well illustrates the vital contribution to physics which may be contained in only a few photographs. Section 6 consists of a single photograph showing the track of a primary cosmic-ray particle in a chamber operated at an altitude of $95,000 \mathrm{ft}$. ; this photograph is indeed $a$ tribute to the skill and ingenuity of the research worker, as well as the reliability of the expansion chamber.

It is to be hoped that the authors intend to keep the material of the book up to date either by appropriate revision or by the publication of a second volume when sufficient progress has been made. On this assumption, a few minor suggestions may be offered. In the technical descriptions of the chambers, more details of the magnets, lamps, cameras and general illumination geometry would be useful. In the case of experiments where the counter selection is complicated, a simple schematic diagram would be valuable; in the first section dealing with technical operation of chambers, more 'faults' should be included showing the different effects of intrinsic chamber faults (for example, inadequate temperature control), control faults, and illumination and/or recording faults.

The price of the present volume is rather high, but it must be appreciated that, where it is required to have an overall reproduction of such a high standard, a high cost is unavoidable. J. R. Atrinson 